\title{
Equations of state for dense and hot matter in compact stars and core collapse supernovae
}

\author{
Micaela Oertel ${ }^{* *}$ \\ LUTH, CNRS-Observatoire de Paris-Université Paris Diderot, 5 place Jules Janssen, 92195 \\ Meudon, France \\ E-mail: micaela.oertel@obspm.fr
}

The properties of compact stars and their formation processes depend on many physical ingredients. The equation of state $(\mathrm{EoS})$ of the involved matter, describing its thermodynamic properties is one of them. It is not an obvious task to construct such an EoS, first of all because very large ranges in baryon number density, temperature and asymmetry have to be covered. Within these ranges the characteristics of matter change dramatically, from an ideal gas of nuclei to uniform strongly interacting matter, containing in the most simple case just free nucleons and potentially many other components such as hyperons, mesons or even quarks. I will summarize existing constraints on the EoS and its composition by terrestrial experiments, astrophysical observations and theoretical considerations. Then I will discuss some recent EoS developments and improvements, with a particular emphasis on the hyperon puzzle, i. e. the fact that many EoSs predict the onset of hyperons at about twice nuclear saturation density and at the same time maximum neutron star masses well below the observed ones.

XIII Nuclei in the Cosmos,

7-11 July, 2014

Debrecen, Hungary

\footnotetext{
* Speaker.

${ }^{\dagger}$ I would like to thank the organizers for invitation and A. Fantina, F. Gulminelli, J. Margueron, J. Novak, B. Peres, A. Raduta for their long-standing collaboration.
} 


\section{Introduction}

The properties of compact stars, their formation as well as binary mergers depend on many different physical ingredients, among them the thermodynamic properties of the involved matter comprised in the equation of state (EoS). There is an intrinsic connection between the properties of matter contained in the EoS for the macroscopic description of astrophysical objects and the underlying fundamental interactions between particles on the microscopic level. This makes the study of the aforementioned systems very rewarding as they challenge our understanding of nature on both scales.

It is not an obvious task to construct such an EoS. The main difficulty arises from the fact that very large ranges of (baryon number) densities $\left(10^{-10} \mathrm{fm}^{-3} \lesssim n_{B} \lesssim 1 \mathrm{fm}^{-3}\right)$, temperatures $(0<T \lesssim 150 \mathrm{MeV})$ and hadronic charge fractions $\left(0<Y_{q}=n_{q} / n_{B} \lesssim 0.7\right)$ have to be covered. $n_{q}$ here denotes the total hadronic charge density, which in many cases is just given by the proton density. Within this range, the characteristics of matter change dramatically, from an ideal gas of different nuclei up to uniform strongly interacting matter, containing in the simplest case just free nucleons and potentially other components such as hyperons, nuclear resonances or mesons. Even a transition to deconfined quark matter cannot be excluded.

For core collapse matter, the full density, temperature, and $Y_{q}$-dependence have to be included within the EoS. This complexity is the main reason why until recently only a few hadronic EoSs existed for core collapse simulations. These are the one by Hillebrandt and Wolff [1], used by some groups performing supernova simulations, that by Lattimer and Swesty [2] and finally that by Shen et al. [3]. The two latter, publicly available, are most commonly used in core-collapse simulations. They use different nuclear interactions, but are based on the same limiting assumptions: they take into account non-interacting $\alpha$-particles, a single heavy nucleus and free nucleons in addition to the electron, positron and photon gas.

Several minutes after their birth, the temperature of neutron stars (and potentially existing quark or hybrid stars) has sufficiently decreased such that for the EoS, matter can be considered as cold. In addition, weak $\beta$-equilibrium is achieved ${ }^{1}$, determining the charge fraction. Therefore those EoS are functions of only one parameter, commonly chosen as $n_{B}$. There is a large variety of EoSs available for cold, dense, $\beta$-equilibrated matter (see for example [4] and references therein), employing different techniques, different nuclear interaction models and different possible compositions, including hyperons, quarks or mesons.

After a brief summary of experimental, theoretical and observational constraints on the EoSs, within this contribution I will highlight some recent developments, for neutron star as well as core collapse and neutron star merger EoS.

\section{Brief summary of experimental, theoretical and observational constraints}

Since dense and hot matter can (presently) not be described from first principles, i.e. starting from the theory of strong interactions, QCD, many uncertainties exist. Most models rely on

\footnotetext{
${ }^{1}$ This means that the reaction $n+v_{e} \rightarrow p+e$ is in equilibrium, thus the respective chemical potentials should fulfill the condition $\mu_{n}+\mu_{v}=\mu_{p}+\mu_{e}$. In a cold neutron star neutrinos can freely leave the system and the corresponding chemical potential thus vanishes, $\mu_{v}=0$.
} 
phenomenological interactions, whose parameters have to be adjusted to existing experimental or observational data. Microscopic many-body calculations (Brueckner-Hartree-Fock, Monte Carlo techniques, renormalisation group, ...) starting from the fundamental two- and three-body forces can to some extent constrain the phenomenological models, too. But since it is impossible to solve the strongly interacting many-body problem exactly, these calculations contain, in addition to the uncertainties on the fundamental forces, more or less controlled approximations and the constraints have to be regarded with some care.

On the experimental side, different coefficients of the Taylor expansion of the energy per baryon of symmetric nuclear matter (i.e. same number of protons and neutrons) can be determined from nuclear experiments. In particular these are the binding energy, the saturation density, the compression modulus and the symmetry energy. It is very challenging to extract other coefficients, such as the slope of the symmetry energy, and the corresponding error bars are very large. From heavy ion collisions, flow data and meson production data, where the analysis within a transport model is reinterpreted as model for the equation of state, can give some indication, too, see [5] for a discussion.

On the astrophysical side, the main present constraint stems from observations of neutron star masses in different binary systems, see e.g. [6] for a compilation. In some of them the masses can be precisely determined from the orbital parameters of the system without much model dependence in the analysis. In particular, precise masses are known for some binary neutron star systems giving masses close to the canonical value of $1.4 M_{\odot}$. Recently, two precise mass determinations in neutron star-white dwarf systems have been carried out. For the first system, the precise determination is based on Shapiro delay, a general relativistic effect, giving a mass of $1.97 \pm 0.04 M_{\odot}$ for the neutron star [7]. The second one combines a well-known model for the white dwarf with an analysis of orbital data to obtain a mass of $2.01 \pm 0.04 M_{\odot}$ for the neutron star [8]. These two solar mass neutron stars are probably not the end of the story since there are indications of even more massive ones in neutron star-brown dwarf systems [9].

For pulsars, the rotation frequency can be determined very precisely, too, but for the moment the fastest known pulsar, PSRJ1748-2446ad, rotates at a frequency of $716 \mathrm{~Hz}$ [10], well below the Kepler frequency for almost all EoS. Thus the constraint induced on the EoS is very weak. An observation of $1.4 \mathrm{kHz}$, on the other hand, would constrain the radius of a non-rotating $1.4 M_{\odot}$ star to be below $9.5 \mathrm{~km}$, very difficult to obtain for most existing EoS.

The ultimate constraint on the EoS would be a determination of radius and mass of the same object, see e.g. [11]. So far, radius observations are, however, much more model dependent than mass measurements. They contain in general different assumptions e.g. on the composition of the atmosphere or the distance of the object and it is difficult to estimate the systematic error on the given values. In addition, for a rotating star due to its deformation there is no unambiguous relation between the observed quantity and the radii determined theoretically.

There are other observations with possible impact on the EoS, but for the moment either the analysis is very model dependent or the observations have large error bars, such that not relevant constraint on the EoS can be obtained for the moment. Examples are the moment of inertia determinations or asteroseismology from the observation of quasi-periodic oscillations. It is of course possible that in the future interesting constraints can come from these studies. Potential gravitational wave observations, in particular of neutron star mergers, could give interesting constraints in 


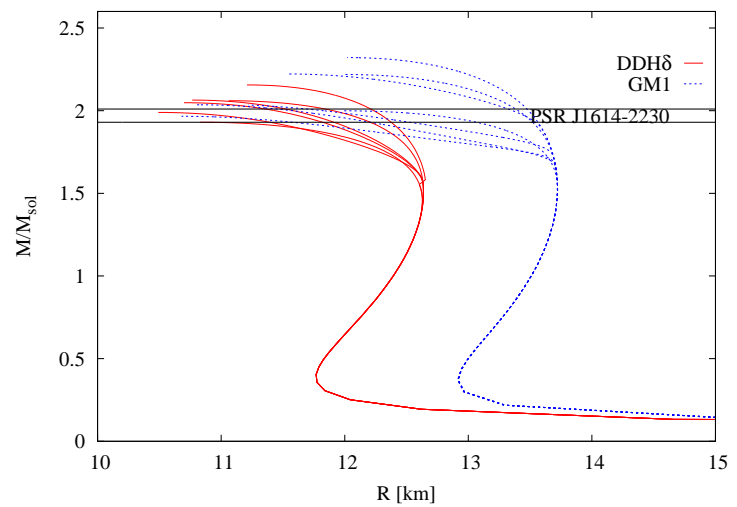

Figure 1: Mass-radius relations for spherically symmetric neutron stars within two relativistic mean field models with different hyperonic interactions.

the future, too $[12,13]$.

\section{Some recent developments}

\subsection{The hyperon puzzle in neutron stars}

The recent discovery of two neutron stars with a mass of about $2 M_{\odot}[7,8]$ triggered intensive discussions about the composition of matter at the center of neutron stars and the EoS at these very high densities. In particular, the so-called "hyperon puzzle" emerged: Most models predict hyperons to appear at $n_{B} \sim 2-3 n_{0}$ but lead at the same time to maximum neutron star masses of $\sim 1.4 M_{\odot}$, well below the highest observed ones. It is thus obvious that additional short-range repulsion is needed to stiffen the high-density EoS.

Different solutions have been proposed to overcome this problem. The first one is that a transition to quark matter appears at densities low enough such that hyperons have not yet softened the EoS too much. The phenomenological quark models can easily be supplemented with the necessary repulsion at high densities, and maximum neutron star masses above $2 M_{\odot}$ can be obtained, see e.g. Refs. $[14,15,16]$.

Another possibility is to modify the hyperonic interactions at high densities. Since experimental data are scarce and furnish therefore only weak constraints on the interactions at densities below nuclear matter saturation density, not much is known about the hyperon-nucleon $(Y N)$ and hyperon-hyperon $(Y Y)$ interactions at the relevant densities in the center of neutron stars. Presently several phenomenological models exist (see e.g. $[17,19,18,20]$ ), containing hyperons in the core and producing maximum neutron star masses in agreement with observations. The crucial point is that the interaction is adjusted to provide the necessary repulsion. As an example, the mass-radius relation for spherically symmetric neutron stars is shown in Fig. 1 for two different relativistic mean field models, varying the hyperonic interaction in agreement with experimental data.

In Brueckner-Hartree-Fock calculations, using a more microscopic approach which starts from a fundamental two- and three body interaction, this seems, however, to be a problem [21,22]. Due to the lack of relevant experimental data on the $Y N$ and $Y Y$ two-body interactions, there are still 
large uncertainties. Hyperonic three-body interactions as input have not yet been fully explored neither and relativistic effects could additionally play a role. The last word is thus not said.

Finally, $\Delta$-resonances could replace hyperons without a transition to quark matter. A first study in this direction leads, however, to a maximum neutron star mass below the observed ones, thus here again the interaction is not sufficiently repulsive [23].

\subsection{Nuclear abundances in core-collapse matter}

In the standard EoS for hot and dense matter [2, 3], the single nucleus approximation (SNA) is used, meaning that not the full distribution of nuclei is taken into account for the EoS, but only one (representative) heavy nucleus and $\alpha$-particles representing light nuclear clusters. Although the nuclear composition is certainly more complicated, the general assumption was that SNA, following [24], is a fair approximation for thermodynamic quantities such as pressure and energy density entering the hydrodynamic equations in the simulations.

Recently, different groups have started to work on improved EoS including the full nuclear distribution. At very low density, a simple nuclear statistical equilibrium approach is sufficient, treating matter as an ideal gas of different clusters neglecting all interactions between them. At higher densities the interaction inside clusters and with the surrounding medium can, however not be neglected, see [25] for a comparison of several different approaches. The conclusion is that the nuclear distribution is very different from SNA. In particular, light clusters other than $\alpha$ particles, such as deutons and tritons can become abundant and the "gap" between "heavy" and "light" clusters is filled up. Except in some small density and temperature regions, the effects on global thermodynamic remains, however, small and the effect on core collapse simulations is visible, albeit not enormous, see e.g. [26]. The effect could be more important on deleptonisation and neutrino interaction rates, see the contribution by T. Fischer to these proceedings.

\subsection{Hot and dense matter at supra-saturation densities}

In stellar core-collapse events and neutron star mergers, matter is heated and compressed to densities above nuclear matter saturation density. The temperatures and densities reached can become so high that a traditional description in terms of electrons, nuclei, and nucleons is no longer adequate. Compared with the cold neutron star EoS, temperature effects favor the appearance of additional particles such as pions and hyperons and they become abundant in this regime.

As an example, in Fig. 2, pressure, energy density and sound speed are shown as functions of temperature for different models, comparing the classical Shen [3] and Lattimer-Swesty [2] EoS to the corresponding versions including additionally $\Lambda$-hyperons [27] or the full baryon octet and pions and muons [20]. All models including additional particles are compatible with experimental data and although the first versions produced too low maximum neutron star masses, now different models exist with a hyperonic interaction that allows for neutron stars in agreement with observations [20,28], see the discussion in the previous section, too.

As can be seen from Fig. 2, the effect of the additional particles on the thermodynamic quantities is not negligible for high density and temperature. During a core collapse such densities and temperatures are reached within the central region, i.e. the contracting proto-neutron star. As a consequence, among others, the time for collapse to a black hole is reduced, see e.g. [29], upon 

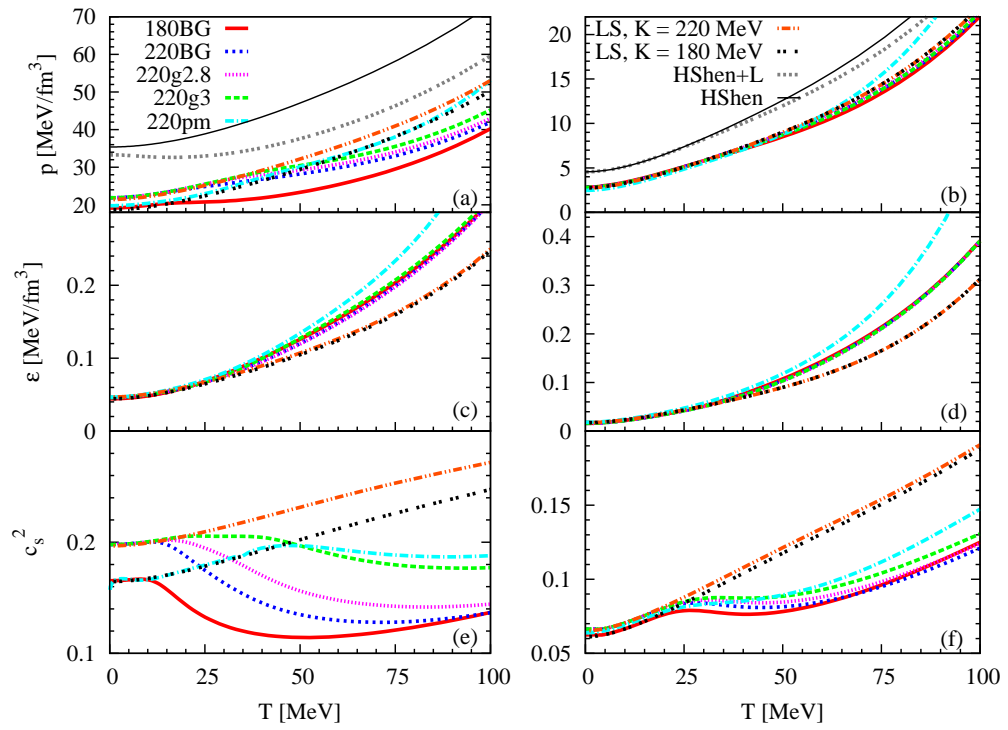

Figure 2: Thermodynamic quantities as functions of temperature for $n_{B}=0.15 \mathrm{fm}^{-3}$ (right) and $n_{B}=$ $0.3 \mathrm{fm}^{-3}$ (left), corresponding roughly to once and twice nuclear matter saturation density, and a charge fraction of $Y_{q}=0.1$. The upper panels show the pressure, the middle ones the internal energy per baryon with respect to the proton mass and the lower ones the sound speed squared. The curves labelled "HShen + L" and "HShen" correspond thereby to the EoS model by Shen et al., including $\Lambda$-hyperons [27] or not [3], respectively. The remaining curves have been calculated within a non-relativistic potential model similar to the LS EoS [2], but containing hyperons, pions and muons, see Ref. [20] for details.

considering hyperons or pions in the EoS. During a neutron star merger, matter is heated up, too, and the change in the EoS due to the additional particles could influence the neutrino and gravitational wave signal $[12,13]$.

\section{Summary and Outlook}

As well as for the description of dense cold matter in neutron stars, much work has been done recently on EoS for hot and dense matter. Concerning the former, the recent observation of two neutron stars with a mass of about $2 M_{\odot}$ has triggered intensive discussion on the composition of matter in the central part of neutron stars and its EoS. In contrast to what has been conjectured in the beginning, these observations do not exclude the existence of other particles than neutrons, protons and electrons in the core. This observation, however, puts stringent constraints on the respective interaction. Different solutions with hyperonic and/or quark matter have been proposed without any definite conclusion.

Concerning the latter, several new models have been constructed, enlarging the variety of nuclear interaction models. This helps to estimate the uncertainty on astrophysical simulations induced by our limited knowledge about the interaction in hot and dense matter. Apart from employing different models, mainly two aspects have been addressed. First, the full nuclear distribution at sub-nuclear densities has been included within different approaches, showing considerable differences to that obtained via the single nucleus approximation in the standard EoS employed in core collapse simulations $[2,3]$. The effect on thermodynamic quantities is small, thus for the moment 
the influence on simulations is not large, but a consistent treatment of deleptonisation and neutrino interaction rates has still to be tested. Second, additional particles have been considered for the high density and temperature part, such as hyperons and mesons or quarks. It has been shown, that this has an influence in particular for black hole formation and in neutron star mergers.

\section{References}

[1] W. Hillebrandt, K. Nomoto, R.G. Wolff, Astronomy \& Astrophysics 133, 175 (1984).

[2] J. M. Lattimer, F. D. Swesty, Nucl. Phys. A 535, 331 (1991).

[3] H. Shen, H. Toki, K. Oyamatsu, K. Sumiyoshi, Nucl. Phys. A 637, 435 (1998).

[4] J. M. Lattimer, M. Prakash, Phys. Rept. 442, 109 (2007).

[5] T. Klahn, D. Blaschke, S. Typel, E. N. E. van Dalen, A. Faessler, C. Fuchs, T. Gaitanos and H. Grigorian et al., Phys. Rev. C 74 (2006) 035802.

[6] J. M. Lattimer and M. Prakash, arXiv:1012.3208 [astro-ph.SR].

[7] P. Demorest et al., Nature 4671081 (2010).

[8] J. Antoniadis, P.C.C. Freire, N. Wex et al., Science, 340, 6131 (2013).

[9] M.H. van Kerkwijk, R.P. Breton, S.R. Kulkarni, Astrophys. J. 728 (2011), 95.

[10] J.W.T. Hessels, S.M. Ransom, I.H. Stairs, P.C.C. Freire, V.M. Kasp et al., Science 311 (2006), 1901.

[11] A.W. Steiner, J.M. Lattimer, E.F. Brown, Astrophys. J. 765 (2013), L5.

[12] Y. Sekiguchi, K. Kiuchi, K. Kyutoku and M. Shibata, Phys. Rev. Lett. 107 (2011) 211101.

[13] A. Bauswein, H. T. Janka, K. Hebeler and A. Schwenk, Phys. Rev. D 86 (2012) 063001.

[14] M. Alford, D. Blaschke, A. Drago, T. Klähn, G. Pagliara and J. Schaffner-Bielich, Nature 445 (2007) E7.

[15] S. Weissenborn, I. Sagert, G. Pagliara, M. Hempel and J. Schaffner-Bielich, Astrophys. J. 740 (2011) L14.

[16] J. L. Zdunik and P. Haensel, Astron. Astrophys. 551 (2013) A61.

[17] I. Bednarek, P.Haensel, J. L. Zdunik, M. Bejger and R. Manka, Astron. Astrophys. 543, A157 (2012).

[18] L. Bonanno and A. Sedrakian, Astron. Astrophys. 529, A16 (2012).

[19] S. Weissenborn, D. Chatterjee, and J. Schaffner-Bielich, Phys. Rev. C 85 (2012) 065802, ibid Nucl. Phys. A881, 62 (2012).

[20] M. Oertel, A. F. Fantina, and J. Novak Phys. Rev. C 85, 055806 (2012); F. Gulminelli, A. R. Raduta, M. Oertel and J. Margueron, Phys. Rev. C 87 (2013) 5, 055809.

[21] I. Vidana, D. Logoteta, C. Providência, A. Polls and I. Bombaci, Europhys. Lett. 94 (2011) 11002.

[22] H.-J. Schulze and T. Rijken, Phys. Rev. C 84 (2011) 035801.

[23] A. Drago et al., arxiv.org:1407.2843.

[24] A. Burrows, J.M. Lattimer, Astrophys. J. 285 (1984) , 294.

[25] N. Buyukcizmeci, A. S. Botvina, I. N. Mishustin, R. Ogul, M. Hempel, J. Schaffner-Bielich, F.-K. Thielemann and S. Furusawa et al., Nucl. Phys. A 907 (2013) 13. 
[26] A. W. Steiner, M. Hempel and T. Fischer, Astrophys. J. 774 (2013) 17.

[27] H. Shen, H. Toki, K. Oyamatsu and K. Sumiyoshi, Astrophys. J. Suppl. 197 (2011) 20.

[28] S. Banik, M. Hempel and D. Bandyopadhyay, Astrophys. J. Suppl. 214 (2014) 2, 22.

[29] B. Peres, M. Oertel and J. Novak, Phys. Rev. D 87 (2013) 4, 043006. 